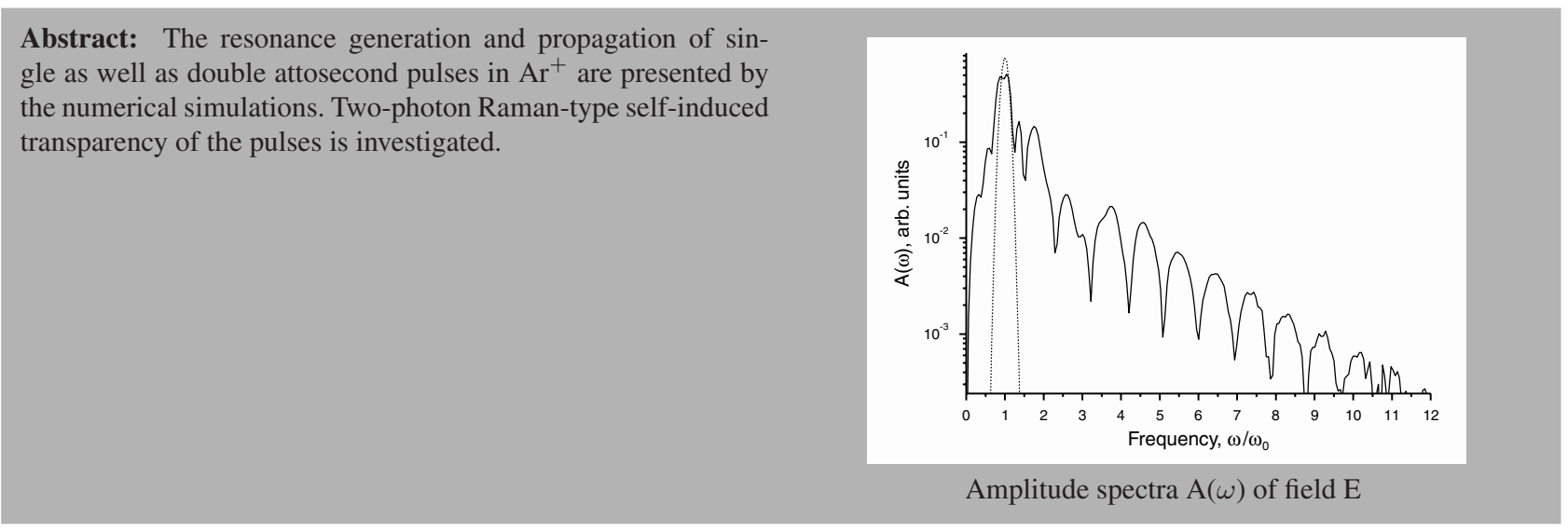

\title{
Single and double attosecond pulses: resonance generation and propagation in $\mathrm{Ar}^{+}$
}

\author{
I.P. Prokopovich* \\ Institute of Nuclear Problems, Belarusian State University, 11 Bobruiskaya Str., 220050 Minsk, Belarus
}

Received: 5 December 2004, Accepted: 12 December 2004

Published online: 11 January 2005

Key words: single attosecond pulses; Argon ions; Raman; two-photon SIT; spectral supercontinuum

PACS: $42.50 . \mathrm{Gy}$, 42.50.Md, 42.65.Dr, 42.65.Re

Much attention is being given to the generation of attosecond pulses (see, for example, [1-5] and references cited therein), using mainly two methods: first, high harmonic generation (HHG) in rare gases; second, high-order stimulated Raman scattering (SRS) at vibration and rotation transitions in molecules, including a solid hydrogen [5]. The advantage of the SRS method is the highenergy conversion efficiency, and the disadvantages are low ionization threshold in comparison with rare gases as well as problems of propagation. For example, the spectrum of a 300-as unipolar pulse covers the spectrum more than $10^{5} \mathrm{~cm}^{-1}(\sim 13 \mathrm{eV})$. Such pulse (and shorter pulses all the more) will capture very many resonant transitions, for example, in $\mathrm{H}_{2}$, that have the largest vibrational transition, the fundamental vibration transition frequency is about $4401 \mathrm{~cm}^{-1}$ (the anharmonicity constant is $121.34 \mathrm{~cm}^{-1}$ ) [6]. As to HHG, the low-energy conversion efficiency $\left(\leq 10^{-3}\right)$ is one of problems, even taking into account methods (see, for example, [4,7-9]) that were proposed recently to increase the efficiency for HHG. Nevertheless, the observation of a train 250 -as pulses that was spaced by 1.35 fs (half the cycle time of the driving 40fs (wavelength of $800 \mathrm{~nm}$ )) from HHG in argon was reported by Paul et al. [10] and Agostini [11]. All above mentioned methods produces discrete spectra and, therefore, only trains of as-pulses. Whereas it is the generation of single attosecond pulses with high intensities that is of prime interest for different applications, including XUV pump-probe experiments on a attosecond time scale. So a substantial effort is being dedicated to generating single attosecond pulses [3-5,12-14].

New way based on electronic stimulated Raman selfscattering (SRSS) of fs-pulses in rare gases to generate intense individual attosecond pulses for the first time was proposed by us [12]. And some possibilities to generate single subfemtosecond pulses from initial $4.63 \mathrm{fs}, 7 \mathrm{fs}$, and $10 \mathrm{fs}$ pulses, using SRSS in singly ionized rare gases, was considered in our previous papers $[1,13]$. SRSS is resonant

\footnotetext{
*Corresponding author: e-mail: prok@inp.minsk.by
} 
coherent SRS when the frequency of Raman transition is captured by spectrum of initial pulse, and first it was investigated at vibration and rotation transitions [15-17]. Continuous Stokes frequency shift with spectral broadening of femtosecond pulses under the condition of SRSS, in vibrational transitions, was experimentally observed in different media [15-16], when initial laser pulse duration $\tau_{0}$ was below 100 fs. In [17-20] it was shown theoretically that under the condition of SRSS the continuous spectral broadening to Stokes direction occurred when the two-photon Rabi frequency $\Omega^{(2)}$, that corresponded to this stimulated Raman process, was less $\pi / \tau_{0}$. But for pulses with more intensity, when the two-photon Rabi frequency was more $\pi / \tau_{0}$ (especially $\sim 2 \pi / \tau_{0}$ ), the superbroadening to both anti-Stokes and Stokes direction, and the generation of spectral supercontinuum occurred [18-20]. In addition, for some conditions a two-photon self-induced transparency (SIT) at SRSS for few-cycle laser pulses had been predicted and an equation for the change in the total energy had been derived [17]. However, the transient pulse behavior was not studied. For the first time, supercontinuum generation in noble gases by fs-pulses was demonstrated by Corkum et al. [21]. Nishioka et al. [22] observed white light, ranging from IR (about $1110 \mathrm{~nm}$ ) to $150 \mathrm{~nm}$, by a self-trapped femtosecond Ti: $\mathrm{Al}_{2} \mathrm{O}_{3}$ laser pulse (125 fs, $790 \mathrm{~nm}, 2$ TW) in atmospheric-pressure Ar. This was the largest frequency coverage continuum as compared with other rare gases. It is necessary to stress that at a laser intensity of about $200 \mathrm{TW} / \mathrm{cm}^{2}$ in argon the tunnelling single ionization time is less than the optical period (the Keldysh parameter equals 0.8 ), and ratio of double to single ionization counts $\mathrm{Ar}^{++} / \mathrm{Ar}^{+}$is less than 0.1 up to pulse intensity of $1000 \mathrm{TW} / \mathrm{cm}^{2}$ [23]. Argon is fully ionized at a laser intensity of about $770 \mathrm{TW} / \mathrm{cm}^{2}$ [24], and the population of Ar neutrals is zero.

Here we report results of the numerical simulations on the resonance generation and propagation of single as well as double attosecond pulses with TW-intensity in $\mathrm{Ar}^{+}$at SRSS by fs-pulses. Two-photon Raman-type self-induced transparency (RSIT) of the pulses are studied.

In $\mathrm{Ar}^{+}$ion the ground state ${ }^{2} \mathrm{P}_{3 / 2}^{o}$ and the neighboring excited state ${ }^{2} \mathrm{P}_{1 / 2}^{o}$ form a two-level system with the frequency transition $\omega_{21} / 2 \pi c=1432 \mathrm{~cm}^{-1}$, and all other levels are removed on $\Delta \geq 108723 \mathrm{~cm}^{-1}$ [6].

Laser pulses with duration $\tau=8$ fs (and shorter), having spectral width $\delta \nu=1 /(c \tau)=4167 \mathrm{~cm}^{-1}$ (and more), satisfies the SRSS condition $[13,15]$

$\delta \equiv \tau \omega_{21} / 2 \pi<1$

where $\hbar \omega_{21}=\epsilon_{2}-\epsilon_{1}$ is the difference in level energies corresponding to the states ${ }^{2} \mathrm{P}_{1 / 2}^{o}$ and ${ }^{2} \mathrm{P}_{3 / 2}^{o}$, as shown in Fig. 1. The laser pulse field $\mathrm{E}$ is described by the wave equation

$\frac{\partial^{2} E}{\partial z^{2}}-\frac{1}{v^{2}} \frac{\partial^{2} E}{\partial t^{2}}=\frac{4 \pi}{c^{2}} \frac{\partial^{2} P_{n l}}{\partial t^{2}}$

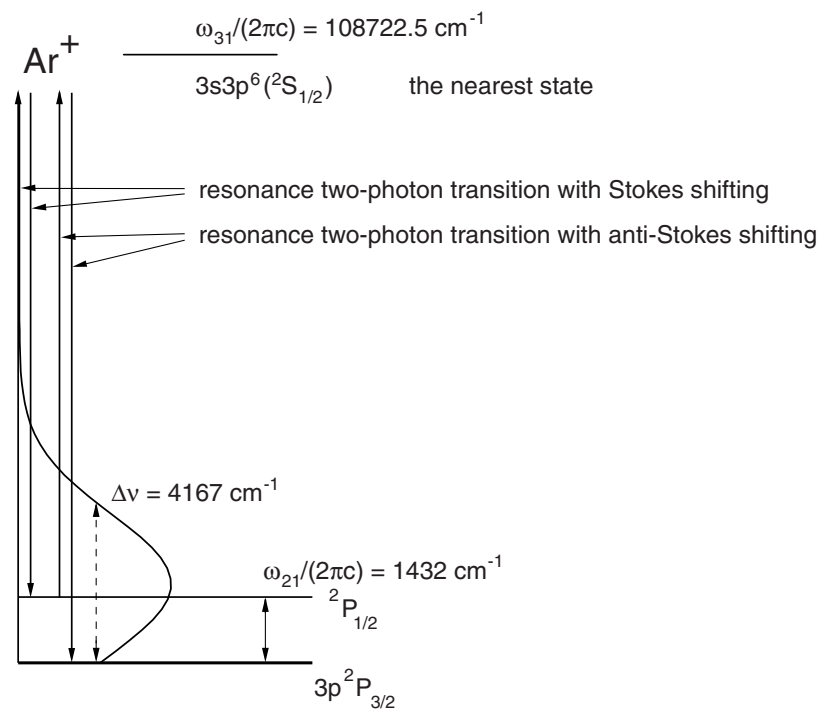

Figure 1 A diagram showing how the SRSS condition is fulfilled for 8-fs laser pulse interacting with $\mathrm{Ar}^{+}$ion being initially in the ground state $3 \mathrm{p}^{2} \mathrm{P}_{3 / 2}^{o}$. The spectrum (Gaussian) of the pulse, having width $\delta \nu=1 /(c \tau)=4167 \mathrm{~cm}^{-1}$, contain the frequency transition $\omega_{21} / 2 \pi c=1432 \mathrm{~cm}^{-1}$

where $v$ is the speed of the laser pulse arising from the linear contribution to polarization, and $P_{n l}$ is the nonlinear contribution to polarization, caused by stimulated Raman process. The direct one-photon transitions between the levels ${ }^{2} \mathrm{P}_{3 / 2}^{o}-{ }^{2} \mathrm{P}_{1 / 2}^{o}$ is forbidden on parity in electrodipole approximation, but permitted for two-photon process [25] like the SRSS. The frequency of Raman transition $\omega_{21}$ is considered to be much less than the central frequency $\omega_{c}$ of the initial laser pulse field $E$. For example, in our case for initial laser pulse with the wavelength of $\lambda=800 \mathrm{~nm}$ the ratio $\omega_{21} / \omega_{c} \simeq 0.1$. The condition of strong two-photon Raman-type resonance is completely satisfied by Eq. (1) for all frequency components $\omega_{1}$ and $\omega_{2}$ of laser pulse when, for example, $\delta \leq 0.5$. The dipole polarizability $\alpha$ in rare gases at two-photon resonance is increased by about 3 orders of magnitude (experimental results show that susceptibility $\chi$ in rare gases at two-photon resonance can be increased by up to 7 orders of magnitude [25] ( $\chi \sim\left|\alpha_{12}\right|^{2}$, where $\alpha_{12}$ is a matrix element of the polarizability corresponding to the Raman transition with frequency $\left.\omega_{21}\right)$. Thus, for laser pulses with duration $\tau=8$ fs and shorter $(\delta \leq 0.34)$ we shall have good approximation (to within one or, at least, a few percents of interaction energy) $\mathrm{V} \simeq-\alpha E^{2} / 2$ (where $\left.\alpha=\alpha\left(\omega_{2}-\omega_{1}=\omega_{21}\right)\right)$ of two-photon interaction Hamiltonian [26,27]. At room temperature and atomic density $\mathrm{N} \leq 3 \times 10^{19} \mathrm{~cm}^{-3}$ the Doppler dephasing time $T_{D}^{*}$, the polarization relaxation time $T_{2}$, and the time of population relaxation $T_{1}$ are estimated from experimental data to be $\left.T_{D}^{*} \simeq 100 \mathrm{ps}, T_{2}>0.2 \mathrm{ps}, T_{2} \ll T_{1}\right)[28,29]$. Hence, first, 
the condition $\tau<T_{2}$ of coherent interaction between the pulse and the two-photon transition is fulfilled very good. Second, the inhomogeneous broadening of the resonance transition can be neglected $\left(\tau / T_{D}^{*}<10^{-3}\right)$. The dynamical Stark shift $\Delta \omega_{s t}=\left(\alpha_{11}-\alpha_{22}\right) E^{2} / 2 \hbar\left(\alpha_{11}, \alpha_{22}\right.$ are values of the diagonal matrix elements of the polarizability) of the resonant levels $\mathrm{Kr} 6 \mathrm{p}[5 / 2]_{2}$ and the nearest upper $6 \mathrm{p}[3 / 2]_{1}$ and lower $6 \mathrm{p}[1 / 2]_{1}$ (about $200 \mathrm{~cm}^{-1}$ ) was estimated as $\Delta \omega_{s t} \leq 40 \mathrm{~cm}^{-1}$ for intensity $\mathrm{I}=1 \mathrm{TW}$ by Kittelmann et al. in their work [29] on direct observation of two-photon coherent response in $\mathrm{Kr}$ by femtosecond UV-laser pulses. It is necessary to note, first, the Pstate in Ar have a value of the dipole polarizability less approximately by a factor of 7.13 as compared to the Pstate in $\mathrm{Kr}$ [6]. Second, a value of the dipole polarizability is decreased very rapidly with decreasing principal quantum number. Third, values of polarizabilities of singly ionized rare gases (in ground state) heavier than He are less than approximately by a factor of 2 as compared to their neutrals [30]. Taking this into account, we can, in our case, to neglect the dynamical Stark shift that connected to the diagonal matrix elements of the polarizability in $\mathrm{Ar}^{+}$(for the $3 p^{5}-{ }^{2} \mathrm{P}_{3 / 2}^{o}$ and $3 p\left({ }^{2} \mathrm{P}_{1 / 2}^{o}\right)$ states ) at least for intensities $\mathrm{I} \leq 10 \mathrm{TW} / \mathrm{cm}^{2}\left(\Delta \omega_{s t}<\omega_{21}\right)$. The nonlinear dipole moment related to SRSS is (see, for example, [31]) $d=\partial V / \partial E=\alpha \cdot E$. Consequently, the nonlinear contribution to the polarization of the medium included in Eq. (2) is $P_{n l}=N \cdot\langle\alpha\rangle \cdot E$, where $N$ is the number density of ions $\mathrm{Ar}^{+}$, and an average value of a polarizability $\langle\alpha\rangle=S p(\alpha \rho)$ can be derived by using the density matrix $\rho$. The function $\left\langle\alpha^{\prime}\right\rangle=\langle\alpha\rangle /\left|\alpha_{12}\right|$ and the difference of the level populations $w=\rho_{22}-\rho_{11}$ are described by the equations $[13,27]$ :

$$
\begin{aligned}
& \frac{\partial^{2}\left\langle\alpha^{\prime}\right\rangle}{\partial t^{2}}+\frac{2}{T_{2}} \cdot \frac{\partial\left\langle\alpha^{\prime}\right\rangle}{\partial t}+\omega_{21}^{2}\left\langle\alpha^{\prime}\right\rangle=-\omega_{21} \Omega^{(2)} \cdot w, \\
& \frac{\partial w}{\partial t}+\frac{\left(w-w_{0}\right)}{T_{1}}=\frac{\Omega^{(2)}}{\omega_{21}}\left(\frac{\partial\left\langle\alpha^{\prime}\right\rangle}{\partial t}+\frac{\left\langle\alpha^{\prime}\right\rangle}{T_{2}}\right),
\end{aligned}
$$

where $\Omega^{(2)}=\left|\alpha_{12}\right| E^{2} / \hbar$ is the two-photon Rabi frequency. For further consideration, it is convenient to introduce an angle of rotation of material variable [17-19]:

$\varphi(t, z)=\frac{\left|\alpha_{12}\right|}{\hbar} \cdot \int_{-\infty}^{t} E^{2}\left(t^{\prime}, z\right) d t^{\prime}$.

The quantity $S(z)=\varphi(+\infty, z)$ is the integral over the entire pulse of the two-photon Rabi frequency of the process. It is easy to see that the $S(z)$ is in direct proportion to total energy of the laser pulse passed the distance $\mathrm{z}$. Value of nonresonant dipole polarizability for Ar in S-state equal to about $1.64 \times 10^{-24} \mathrm{~cm}^{3}$, and in P-state equal to about $7.1 \times 10^{-24} \mathrm{~cm}^{3}$ [6]. As noted above, values of polarizabilities of singly ionized rare gases (in ground state) heavier than $\mathrm{He}$ are less than approximately by a factor of 2 as compared to their neutrals. For example, ion $\mathrm{Ne}^{+}$ and atom Ne have the values of the static polarizabilities $0.21 \times 10^{-24} \mathrm{~cm}^{3}$ and $0.395 \times 10^{-24} \mathrm{~cm}^{3}$, respectively [30]. So with a knowledge of the dynamic polarizability for $\mathrm{Ar}$ at $800 \mathrm{~nm}$, and taking into account above-mentioned increasing its at two-photon resonance, the value of the matrix element of the polarizability $\left|\alpha_{12}\right|$ (corresponding to the transition ${ }^{2} \mathrm{P}_{3 / 2}^{o}-{ }^{2} \mathrm{P}_{1 / 2}^{o}$ in $\mathrm{Ar}^{+}$) can be estimated to be in range $10^{-21} \div 10^{-20} \mathrm{~cm}^{3}$.

The numerical solutions of wave equation (2) with Eqs. (3) and (4) in the case of a coherent interaction for a laser pulse when $S(0)=2 \pi \cdot n(n=1,2,3)$ and for various $\beta \cdot z \leq 2$, where $\beta=2 \pi N\left|w_{0}\right| \cdot\left|\alpha_{12}\right| \omega_{21} / c\left(N \leq 3 \times 10^{19} \mathrm{~cm}^{-3}\right)$, had shown that no more than one per cent (within $1 \%$ accuracy of the simulation) of the pulses energy was scattered in the reverse direction [13]. [The quantity $\beta^{-1}$, which has the dimension of length, can be interpreted as the length of coherent SRSS.] It is therefore a very good approximation to introduce a reduced wave equation $[13,17-20]$ :

$\frac{\partial E}{\partial z}+\frac{1}{v} \frac{\partial E}{\partial t}=-\frac{4 \pi v}{c^{2}} \frac{\partial P_{n l}}{\partial t}$,

which describes only the forward scattering of the field. First the reduced wave equation was derived by Bullough, Eilbek et al. for one-photon SIT [32]. If a dimensionless constant

$\alpha_{s}^{(1)}=\frac{4 \pi N d_{21}^{2}}{\hbar \omega_{21}}$

(where the $N$ is an atomic density, the $d_{21}$ is a matrix element of the one-photon resonant transition with frequency $\left.\omega_{21}\right)$ is less than 1 then the reduced Maxvell-Bloch equations (the Maxvell-Bloch equations in which only backscattering is neglected) provides good description SIT for pulses with duration up to half-cycle of the electromagnetic field. For example, for a typical value of $d_{21}, 10^{-18}$ cgs units, this is a good approximation (to within 1\%) at $N=10^{18} \mathrm{~cm}^{-3}$. It should be stressed that the numerical simulations using Eq. (6) have significantly less expenses of computer time as compared with Eq. (2). In addition, when $\delta \ll 1$, the laser pulse energy is altered by the law $[17,19]$

$\frac{d S}{d z}=\beta(1-\cos S)$,

which is practically the same as for the case of a coherent direct two-photon interaction of a field with a resonant medium, that was first derived by Belenov and Poluektov [33]. This is area theorem for two-photon self induced transparency. Such laser pulses with $S(0)=2 \pi n$ can propagate through medium $(\beta \cdot z \approx 1)$ in the RSIT regime [17-20]. Furthermore, the propagation of the pulses with $S=2 \pi[12,13,18,20], S=4 \pi[13]$, and $S=6 \pi$ [34] leads to effective self-broadening of the spectrum and the generation of a spectral supercontinuum due to the SRSS.

The dynamics of a laser pulse having initially a Gaussian shape given by

$E(t, 0)=E_{0} \sin \left(\omega_{c} t\right) \exp \left\{-2 \ln 2 \cdot\left(\frac{t}{\tau_{0}}\right)^{2}\right\}$, 
where $E_{0}$ is the initial peak field amplitude of the pulse propagating through the medium unexcited initially (i.e., $\left.\left(w(0, z)=w_{0}=-1,\langle\alpha(0, z)\rangle=0\right)\right)$, is investigated numerically by the Eqs. (3), (4), and (6). An improved and many times tested version of the code presented in $[12,13,17-20]$ is used on grid of 4096 points per one period of an electromagnetic field. This provided good solution (better than $1 \%$ accuracy). As far as we know, there are no direct experimental data on the dynamic polarizability or the two-photon Rabi frequency regarding the two-photon Raman-type resonance at the transition ${ }^{2} \mathrm{P}_{3 / 2}^{o}-{ }^{2} \mathrm{P}_{1 / 2}^{o}$ in $\mathrm{Ar}^{+}$to estimate directly the value of the matrix element of the polarizability $\left|\alpha_{12}\right|$. Taking into account the above estimations of the $\left|\alpha_{12}\right|$ we performed the numerical simulations for the broad range of the parameter $\gamma=2 \pi N\left|\alpha_{12}\right| \simeq 0.04 \div 0.2$, and we found similar results, but as some function of $\beta \cdot z$. These results and an extended discussion of this subject will be published in next work.

In Fig. 2 we show the dynamics of the field $E$ of an initial three-cycle (within FWHM) laser pulse $(\beta z=0)$ (Fig. 2a) when $\gamma=0.2, S(0)=6 \pi$, and its field at $\beta z=0.68$ (Fig. 2b). The dynamics of its spectrum is illustrated in Figs. $3 \mathrm{a}$ and $3 \mathrm{~b}$, respectively. This $6 \pi$-pulse propagated a distance corresponding to $\beta z=0.68$ in regime of RSIT, conserving its energy about $98 \%$ (to within $1 \%$ calculation). The initial $6 \pi$-pulse have splitting into three $2 \pi$-pulses (Fig. 2b), that with conserved total energy of the initial pulse are unique signatures of self-induced transparency. Some properties of the two-photon Raman-type self-induced transparency for few-cycle laser pulses were studied recently [34]. In particular, it was shown that after such splitting of an initial six-cycle (FWHM) $6 \pi$-pulse into three $2 \pi$-pulses the first and second $2 \pi$-pulses was transformed into one-cycle (FWHM) pulses greater amplitudes and photon energies (see Figs. 5 and 6 of [34]). Fig. 2b shows, in case of an initial three-cycle (FWHM) $6 \pi$-pulse, the first and second $2 \pi$-pulses are transformed into halfcycle (FWHM) pulses greater amplitudes and photon energies. Field lengthes of the $2 \pi$-pulses are decreased and at the $\beta z=0.68$ ones are $\tau_{1}=0.14 T_{0}$ and $\tau_{2}=0.15 T_{0}$ (FWHM), where $T_{0}$ is a period of one oscillation of the initial field $\mathrm{E}\left(T_{0}=2 \pi / \omega_{c}\right)$. Time distance between these pulses is $\tau_{21}=1.12 T_{0}$ (FWHM). For example, if an initial three-cycle laser pulse have wavelength $\lambda=800 \mathrm{~nm}$ then $T_{0}=2.667 \mathrm{fs}, \tau_{0}=8 \mathrm{fs}, \tau_{1}=0.37 \mathrm{fs}, \tau_{2}=0.40 \mathrm{fs}$, and $\tau_{21}=2.99$ fs. Dynamics of amplitude spectrum $\mathrm{A}(\omega)$ of the field E corresponding to Fig. 2 is shown in Fig. 3.

Because, at present, the experimental technique to generate two-cycle (FWHM) laser pulses with TW-intensity is developed [4] we investigated a feasibility to generate a single attosecond pulse from such two-cycle pulses. Our numerical simulation shown that one of the best results can be obtained if to use the initial two-cycle laser pulses with $S(0)=2.7 \pi$. Thus the pulses are not propagated initially in the RSIT regime. However, after some distance $(\beta z \simeq 1.5)$ they will have $S(z) \simeq 2 \pi$ and propagate under the RSIT conditions. The results with the two-

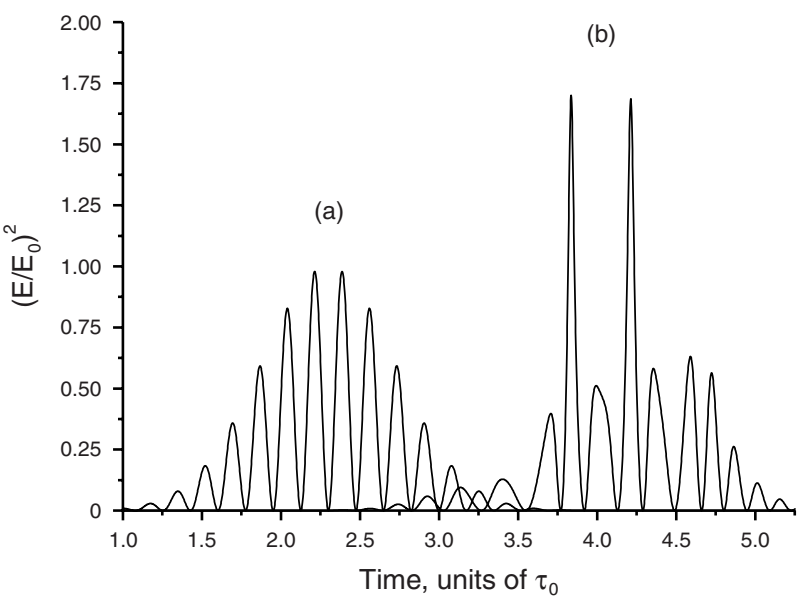

Figure 2 Dynamics of squared field E (normalized to initial amplitude $E_{0}$ ) vs. time (normalized to initial pulse width $\tau_{0}$ ) for an initial three-cycle (FWHM) laser pulse (a) $(\beta z=0), S(0)=6 \pi$, $\gamma=0.2$, and (b) $\beta z=0.68$

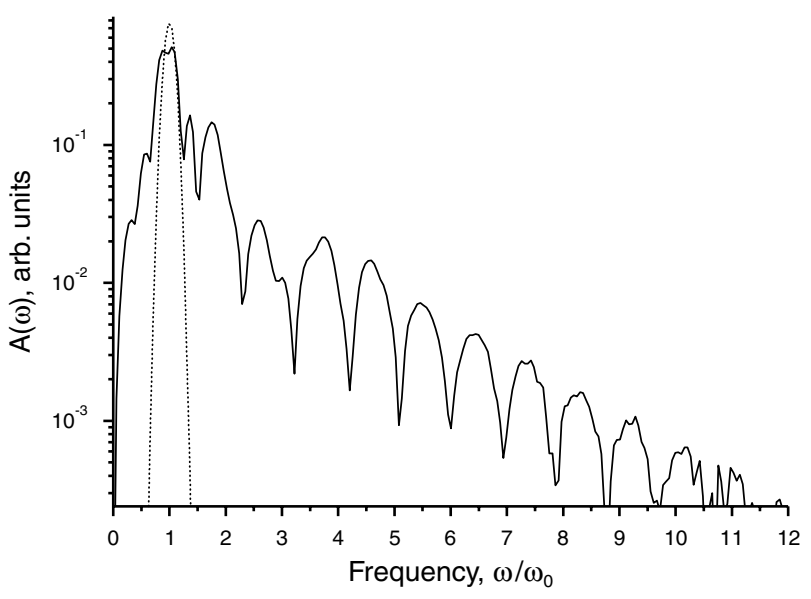

Figure 3 Amplitude spectra $\mathrm{A}(\omega)$ of field $\mathrm{E}$, corresponding to Fig. 2. The dotted line is spectrum of initial field $(\beta z=0)$. The firm line is spectrum of field after distance corresponding to $\beta z=0.68$

cycle (FWHM) input pulse are presented in Fig. 4, and the corresponding spectra are shown in Fig. 5. Passing some distance corresponding to $\beta z \simeq 1.5$ the two-cycle pulse (Fig. 4a) is transformed into the half-cycle pulse (FWHM) (Fig. 4b) with the duration $\tau=\tau_{0} / 16=0.125 T_{0}$. The spectrum of its field is smooth supercontinuum (Fig. 5) as compared with one of the double half-cycle pulses (Fig. 3). For example, if an initial two-cycle laser pulse have wavelength $\lambda=800 \mathrm{~nm}$ then $T_{0}=2.667 \mathrm{fs}, \tau_{0}=5.33$, and $\tau=0.33$ fs.

Using the foregoing parameters of the numerical simulations we can give, as an example, some correspond- 


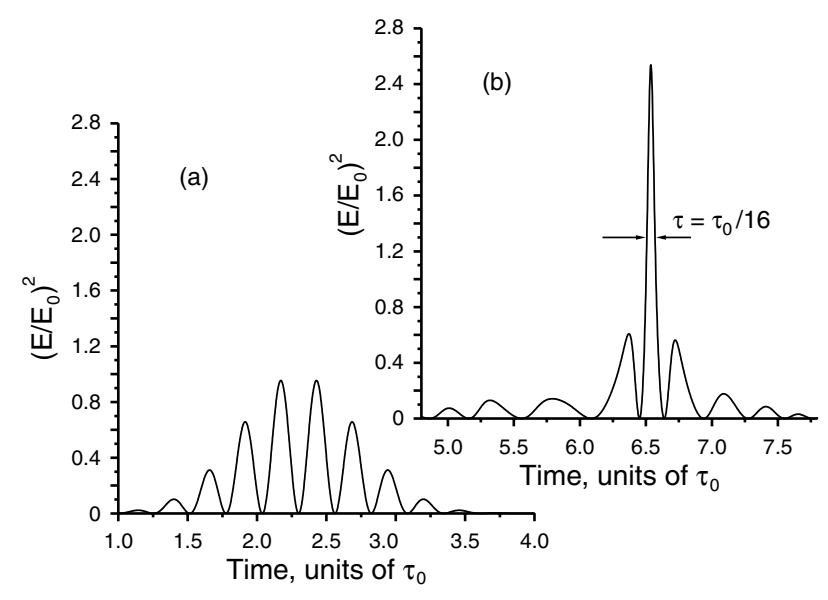

Figure 4 Dynamics of squared field E (normalized to initial amplitude $E_{0}$ ) vs. time (normalized to initial pulse width $\tau_{0}$ ) for an initial two-cycle (FWHM) laser pulse (a) $(\beta z=0)$, $S(0)=2.7 \pi, \gamma=0.2$, and (b) $\beta z=1.5$

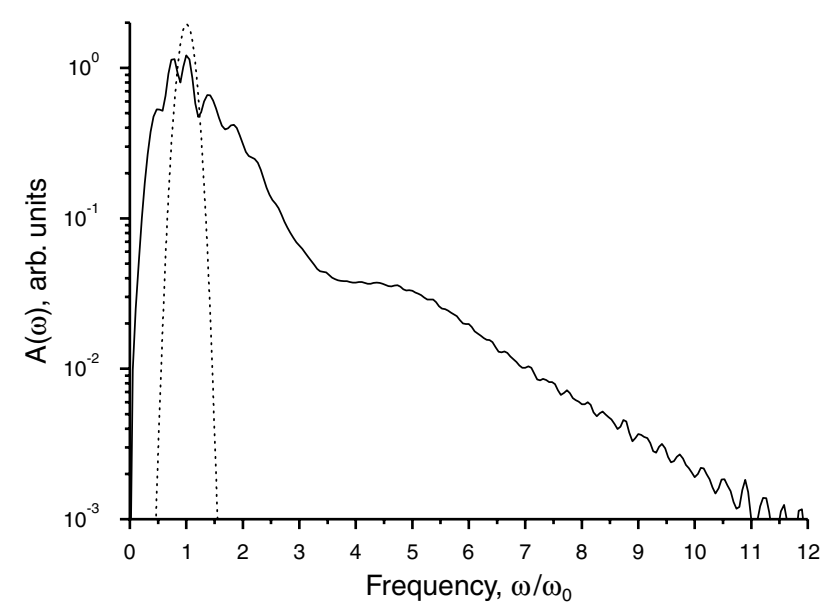

Figure 5 Amplitude spectra $\mathrm{A}(\omega)$ of field $\mathrm{E}$, corresponding to Fig. 4. The dotted line is spectrum of initial field $(\beta z=0)$. The firm line is spectrum of field after distance corresponding to $\beta z=1.5$

ing concrete parameters for future experimental investigations. It is easy to see that $\gamma \simeq 0.2$ and $\beta \simeq 1.8 \times 10^{3} \mathrm{~cm}^{-1}$ if, for example, $N=3 \times 10^{18} \mathrm{~cm}^{-3}$ and $\left|\alpha_{12}\right|=$ $10^{-20} \mathrm{~cm}^{3}$ or $N=3 \times 10^{19} \mathrm{~cm}^{-3}$ and $\left|\alpha_{12}\right|=10^{-21} \mathrm{~cm}^{3}$. For 5-fs two-cycle laser pulse and $\left|\alpha_{12}\right|=3 \times 10^{-21} \mathrm{~cm}^{3}$ we will have $S(0) \simeq 3 \pi$ at $\mathrm{I} \simeq 10^{12} \mathrm{~W} / \mathrm{cm}^{2}$, and the propagation distances $\mathrm{z} \simeq 8 \mu \mathrm{m}$ as regard Fig. 4b. In addition, using the same method as in [34] we have calculated the effect of the plasma on coherent lengths. For example, at densities $N=10^{19} \mathrm{~cm}^{-3}$ of ions $\mathrm{Ar}^{+}$and free electrons as well as for the initial 5-fs two-cycle laser pulse with $\lambda=800 \mathrm{~nm}$, this coherent length $L_{c} \simeq 12 \mu \mathrm{m}$ for the 330-attosecond pulse (Fig. 4b) having the spectral supercontinuum corresponding to Fig. 5.

In summary, we have shown that the three- and twocycle laser pulses, having the length less than $10 \mathrm{fs}$, can be transformed efficiently into double and single halfcycle attosecond pulses, respectively, in $\mathrm{Ar}^{+}$by a resonant two-photon Raman-type transitions. I addition, these pulses having TW-range peak intensities can propagate some distances in regime of the two-photon Raman-type self-induced transparency. The coherent lengths, regarding plasma mismatching, have been calculated and are less than the generation lengths of the attosecond pulses.

Acknowledgements This work was partially supported by the B.I.Stepanov Institute of Physics NASB, "Coherence" Program (grant "Coherence-62"), by the BSU, and by the ISTC Project B-1065.

\section{References}

[1] T.W. Hänsch, Opt. Comm. 80, 71 (1990); S.M. Gladkov and N.I. Koroteev, Sov. Phys. Usp. 33, 554 (1990).

[2] G. Farkas and C. Toth, Phys. Lett. A168, 447 (1992); S.E. Harris, J.J. Macklin, and T.W. Hänsch, Opt. Comm. 100, 487 (1993); S. Yoshikawa and T. Imasaka, Opt. Comm. 96, 94 (1993); A.E. Kaplan, Phys. Rev. Lett. 73, 1243 (1994); S.E. Harris and A.V. Sokolov, Phys. Rev. Lett. 81, 2894 (1998).

[3] P.B. Corkum, N.H. Burnett, and M.Y. Ivanov, Opt. Lett. 19, 1870 (1994); M. Ivanov, P.B. Corkum, T. Zuo, and A. Bandrauk, 74, 2933 (1995); P. Antoine, A. L'Huillier, and M. Lewenstein, Phys. Rev. Lett. 77, 1234 (1996); I.P. Christov, M.M. Murnane, and H.C. Kapteyn, Phys. Rev. Lett. 78, 1251 (1997).

[4] T. Brabec and F. Krausz, Rev. Mod. Phys. 72, 545 (2000).

[5] N.H. Shon, F.L. Kien, K. Hakuta, and A.V. Sokolov, Phys. Rev. A 65, 033809 (2002).

[6] A.A. Radzig and B.M. Smirnov, Reference Data on Atoms, Molecules and Ions (Heidelberg, Springer, 1985).

[7] Z. Chang, A. Rundquist, H. Wang, et al., Phys. Rev. Lett. 79, 2967 (1997); M. Schnurer, Ch. Spielmann, P. Wobrauschek, et al., Phys. Rev. Lett. 80, 3236 (1998); Y. Tamaki, J. Itatani, Y. Nagata, et al., Phys. Rev. Lett. 82, 1422 (1999); G. Tempea, M. Geissler, M. Schnürer, and T. Brabec, Phys. Rev. Lett. 84, 4329 (2000); R. Baitels at al., Nature 406, 164 (2000).

[8] J.B. Peatross, S. Voronov, and I. Prokopovich, Opt. Expr. 1, 114 (1997); S.L. Voronov, I. Kohl, J.B. Madsen, et al., Phys. Rev. Lett. 87, 133902 (2001).

[9] I.P. Prokopovich, A.A. Khrutchinsky, D.Yu. Churmakov, et al., Phys. Rev. A 65, 053823 (2002); I.P. Prokopovich, A.A. Khrutchinsky, D.Yu. Churmakov, Proc. SPIE 4752, 167 (2002).

[10] P.M. Paul, E.S. Toma, P. Breger, et al., Science 292, 1689 (2001).

[11] P. Agostini and P.M. Paul, Proc. SPIE 4752, 136 (2002).

[12] I.P. Prokopovich, Generation of Attosecond Pulses in the Raman Self-Scattering of High-Intense Femtosecond Optical Pulses, in: Proceedings of the 5th International Workshop on Laser Physics, Moscow, 1996, p. 15. 
[13] I.P. Prokopovich and A.A. Khrushchinskii, Laser Phys. 7, 305 (1997); I.P. Prokopovich and J. Peatross, Laser Phys. 9, 588 (1999); I.P. Prokopovich and J. Peatross, Proc. SPIE 4752, 31 (2002).

[14] M. Drescher, M. Hentschel, R. Kienberger, et al., Science 291, 1923 (2001); M. Hentschel, R. Kienberger, C. Spielmann, et al., Nature 414, 509 (2001); M. Drescher, M. Hentschel, R. Kienberger, et al., Nature 419, 803 (2002); A. Baltuska, Th. Udem, M. Uiberacker, et al., Nature 421, 611 (2003).

[15] S.A. Akhmanov, V.A. Vyslouch, and A.S. Chirkin, Optics of Femtosecond Laser Pulses (AIP, New York, 1992).

[16] E.M. Dianov, A.Ya. Karasik, V.P. Mamyshev, et al., JETP Lett. 41 (1985); Y.X. Yan, E.B. Gamble, Jr., and K.A. Nelson, J. Chem. Phys. 83, 5391 (1985); S. De Silvestri, J.G. Fujimoto, E.P. Ippen, et al., Chem. Phys. Lett. 116, 146 (1985); F.M. Mitchke and L.F. Mollenauer, Opt. Lett. 11, 659 (1986); A.B. Grudinin, E.M. Dianov, D.V. Korobkin, et al., JETP Lett. 45 (1987).

[17] E.M. Belenov, A.V. Nazarkin, and I.P. Prokopovich, Pis'ma Zh. Eksp. Teor. Fiz. 55, 223 (1992) [JETP Lett. 55, 218 (1992)].

[18] E.M. Belenov and I.P. Prokopovich, Kvant. Electron. 20, 577 (1993) [Quantum Electron. 23, 497 (1993)].

[19] E.M. Belenov, P.G. Kryukov, A.V. Nazarkin, and I.P. Prokopovich, Zh. Eksp. Teor. Fiz. 105, 28 (1994) [JETP 78, 15 (1994)].

[20] E.M. Belenov and I.P. Prokopovich, J. Russ. Laser Research 15, 283 (1994).

[21] P.B. Corkum, C. Rolland, and T. Srinivasan-Rao, Phys. Rev.
Lett. 57, 2268 (1986); P.B. Corkum and C. Rolland, IEEE J. Quant. Electron. 25, 2634 (1989).

[22] H. Nishioka, W. Odajima, Ken-ichi Ueda, H. Takuma, Opt. Lett. 20, 2505 (1995).

[23] J.L. Chaloupka, J. Rudati, R. Lafon, et al., Phys. Rev. Lett 90, 033002 (2003).

[24] E.A. Gibson, A. Paul, N. Wagner, et al., Phys. Rev. Lett 92, 033001 (2004).

[25] J.F. Reintjes, Nonlinear Optical Parametric Processes in Liquids and Gases (Academic Press, London, 1984).

[26] Y.R. Shen, The Principles of Nonlinear Optics (Wiley, New York, 1984).

[27] R.H. Pantell and H.E. Puthoff, Fundamentals of Quantum Electronics (Wiley, New York, 1969).

[28] R.W. Boyd, Nonlinear Optics (Academic Press, London, 1992).

[29] O. Kittelmann, J. Ringling, A. Nazarkin, et al., Phys. Rev. Lett. 76, 2682 (1996).

[30] E.W. McDaniel and E.A. Mason, The mobility and diffusion of ions in gases (Wiley, New York, 1973).

[31] L.D. Landau and E.M. Lifshits, Quantum Mechanics (Pergamon, Oxford, 1977).

[32] R.K. Bullough and F.Ahmad, Phys. Rev. Lett. 27, 330 (1971); J.C. Eilbeck, J.D. Gibbon, P.J. Caudrey, and R.K.Bullough, J. Phys. A 6, 1337 (1973); P.J. Caudrey, J.D. Gibbon, and J.C. Eilbeck, Phys. Rev. Lett. 30, 237 (1973).

[33] E.M. Belenov and I.A. Poluektov, Zh. Eksp. Teor. Fiz. 56, 1407 (1969)[JETP 29, 754 (1969)].

[34] I.P. Prokopovich, Laser Phys. Lett. 2, 120 (2005). 